\title{
Aprendizaje ambiental profundo en la UPV
}

\author{
Inmaculada Romero ${ }^{\mathrm{a}}$, Maria Pachés ${ }^{\mathrm{a}}, \mathbf{M}^{\mathrm{a}}$ Teresa Sebastiáb y Carmen Hernández-Crespo $^{\mathrm{a}}$ \\ a Escuela Técnica Superior de Ingeniería de Caminos, Canales y Puertos (Universitat Politècnica de València) \\ inrogi@upv.es, y ${ }^{\text {b }}$ Escuela Politécnica Superior de Gandia (Universitat Politècnica de València)
}

\begin{abstract}
Within the framework of the Sustainable Development Goals (SDGs), our general objective is to improve the quality of student learning, from the point of view of sustainable development and to achieve deep learning in this area. The active learning methodology used (Project Based Learning) has made our students learn collaboratively and cooperatively, promoting their motivation and achieving deep learning in environmental aspects.

The results showed that students use more and better learning strategies. Motivational strategies improved, confirming that the use of applied methodology improves the motivation of students. Metacognitive strategies also improve greatly, which is highly related to the work required of students who need this type of skills. In the same way, they also improve all the strategies related to the processing of information, which are essential to successfully carry out learning. It has been achieved that students learn better, both individually and in groups, with an active, constant and cooperative participation, taking more responsibility in the development of their work and getting closer to the reality of their professional future.
\end{abstract}

Keywords: Sustainable Development Goals, Deep Learning, CEVEAPEU, Project Based Learning

\section{Resumen}

En el marco de los Objetivos de Desarrollo Sostenible (ODS), nuestro objetivo general es mejorar la calidad del aprendizaje de los estudiantes, desde el punto de vista del desarrollo sostenible y que alcancen un aprendizaje profundo en este ámbito. La metodología de aprendizaje activa utilizada (Aprendizaje Basado en Proyectos), ha conseguido que nuestros estudiantes aprendan de manera colaborativa y cooperativa, fomentando su motivación y logrando que alcancen un aprendizaje profundo en aspectos medioambientales.

Los resultados demostraron que los estudiantes utilizan más y mejores estrategias de aprendizaje. Las estrategias Motivacionales mejoraron, lo que confirma que el uso de la metodología aplicada hace mejorar la motivación de los estudiantes. También las estrategias Metacognitivas mejoran en gran medida, lo que está altamente relacionado con el trabajo exigido a los estudiantes, que necesitan este tipo de habilidades. Del mismo modo también mejoran todas las estrategias relacionadas con el procesamiento de la información, que son imprescindibles para llevar a cabo con éxito el aprendizaje. Se ha conseguido que los estudiantes aprendan mejor, tanto de forma individual como en grupo., con una participación activa, constante y cooperativa, teniendo más responsabilidad en el desarrollo de su trabajo y acercándose a la realidad de su futuro profesional.

Palabras clave: Objetivos de Desarrollo Sostenible, Aprendizaje profundo, CEVEAPEU, Aprendizaje Basado en Proyectos 


\section{Introducción}

La Agenda 2030 para el Desarrollo Sostenible adoptada por la ONU tiene como objetivo global favorecer a las personas, al planeta y a su prosperidad. Plantea 17 Objetivos de Desarrollo Sostenible (ODS) formulados con el fin de erradicar la pobreza, promover la prosperidad y el bienestar, proteger el medio ambiente y hacer frente al cambio climático. Los 17 ODS definidos conllevan 169 metas o aspiraciones a nivel mundial, aunque cada gobierno ha fijado sus metas propias, sugiriendo además cómo incluirlas en sus procesos de planificación, políticas y estrategias nacionales. Aunque los 17 ODS, están íntimamente relacionados con la sostenibilidad (económica, social y ambiental), en este trabajo nos centramos sobre los ODS relacionados con el medio ambiente (biosfera), concepto transversal que está incluido en los ODS (Rockström \& Sukhdev, 2016).

En España, el gobierno español, define claramente a las Universidades como facilitadoras y actores clave para promover el desarrollo humano sostenible, fundamentalmente debido a la responsabilidad que tienen en la educación, en el desarrollo del espíritu crítico y en la incorporación de los principios y valores del desarrollo sostenible (Ministerio de Asuntos Exteriores, 2018). De hecho, se incorporaron en todas las titulaciones universitarias las competencias en sostenibilidad, siendo en la UPV la "CT 07. Responsabilidad ética, medioambiental y profesional”. Incluso el Reto 5 del Plan Estratégico de la UPV, UPV2020, incluye "Destacar por sus compromisos en materia de responsabilidad social como universidad pública". Dentro del Proyecto 5.4. Sostenibilidad ambiental, se incluye el objetivo 5 (RE5P4O5), "Ser una Universidad influyente en su entorno mediante la transmisión de los valores Ambientales".

Entwistle (2009) en sus trabajos sobre los enfoques de aprendizaje, concluye que los estudiantes tienden a aprender en función de las tareas que se les solicite. Pueden simplemente memorizar y posteriormente reproducir (enfoque de aprendizaje superficial) o pueden llegar a aprender de manera significativa (enfoque de aprendizaje profundo). Lo deseable es desarrollar este segundo enfoque a través del diseño de actividades de aprendizaje que supongan retos para los estudiantes. Así, evaluar las estrategias y enfoques de aprendizaje de los estudiantes puede dar un diagnóstico para implementar medidas pedagógicas para su mejora (Fernandez \& Arquero, 2011).

Generalmente, un enfoque de aprendizaje superficial se relaciona con una falta de motivación a aprender, lo que a su vez da lugar a una disminución de la motivación. Sin embargo, un enfoque profundo se relaciona con sujetos altamente motivados, que genera en muchas ocasiones un aumento de la motivación (DíazMújica \& Pérez-Villalobos, 2013). La estrategia profunda suele establecerse en base a una motivación intrínseca, orientada a querer saber, mientras que en la estrategia superficial la motivación suele ser externa, orientada a aprobar (Fasce, 2007).

Es un error considerar que debe evitarse el aprendizaje superficial en los estudiantes. Ambos enfoques son necesarios, pues si se quiere promover aprendizajes profundos previamente deben aprenderse conocimientos básicos y fundamentales que posteriormente se utilizarán para comprender y reflexionar sobre un tema concreto de forma profunda. Además, un estudiante no tiene porqué enfocar su aprendizaje de la misma manera en todas las asignaturas, ni siquiera en todos los temas de cada asignatura, depende de cómo considere la tarea que se le pide. De hecho, se considera que los enfoques de aprendizaje de un mismo estudiante van cambiando conforme se van encontrando con los diferentes tipos de enseñanza y de tareas que se les solicitan (Entwistle, 2009; Fernández \& Arquero, 2011; Díaz-Mújica \& Pérez-Villalobos, 2013). Así, no puede decirse que existan estudiantes superficiales y estudiantes profundos, pues éstos pueden modificar su enfoque o forma de aprender en función de las necesidades de cada momento (López-Aguado \& López-Alonso, 2013; Fasce, 2007). Por ello, en cada disciplina, o incluso en cada tarea, se debe definir cuál es la mejor manera de potenciar el aprendizaje profundo. 
Generalmente sobrecargar al estudiante de contenidos y evaluarlo de manera continua con pequeños exámenes o pruebas memorísticas y sin conexión clara entre los temas, e incluso sin feedback, favorece que los estudiantes adopten un enfoque superficial. Sin embargo, como apuntan Biggs \& Tang (2007) y Ramsden (2003), si lo que se desea es fomentar el aprendizaje profundo debe establecerse claramente qué expectativas tiene el estudiante y qué conocimientos previos posee sobre el tema. Se deben plantear actividades que fomenten la participación activa y que les ayuden a realizar conexiones entre diferentes temas, cursos y disciplinas. Incluso en ocasiones puede ser bueno dar oportunidades a los alumnos respecto a la selección de temas o sistemas de evaluación. No hay que olvidar la importancia de proporcionar feedback a los estudiantes sobre los aspectos que necesitan mejorar y sobre sus fortalezas. Con ello generalmente se conseguirá además un mayor interés y motivación del estudiante en la materia (Fasce, 2007). De hecho, se ha visto que la estrategia de enseñanza que se emplee orientará el aprendizaje de diferente manera y por lo tanto los aprendizajes resultantes también diferirán [Fernández \& Arquero, 2011; Díaz-Mújica \& Pérez-Villalobos, 2013; Antonelli, 2017; López-Aguado \& Gutierrez-Provecho, 2014).

El Aprendizaje Basado en Proyectos (ABP) está siendo ampliamente utilizado como estrategia de aprendizaje profundo. Es una estrategia que aumenta la motivación de los estudiantes, pues permite en primer lugar que ellos mismos seleccionen los temas que más les interesen y que serán importantes en su futuro profesional (Castro et el., 2018; Maldonado, 2008; Rodríguez-Sandoval et al., 2010; Katz \& Chard, 1989). Los estudiantes trabajan de manera activa, plantean el proyecto, trabajan de manera colaborativa, toman decisiones, lo implementan y lo evalúan. Una de sus características principales es que está orientado a la acción. Como apuntan Martí et al. (2009) el docente actúa como un orientador o guía, porque es una herramienta que se centra en el estudiante, promoviendo la motivación intrínseca. Además, estimula el aprendizaje colaborativo y cooperativo. Generalmente muchos de los Proyectos que se plantean se conciben como la búsqueda de una solución al planteamiento de un caso concreto del mundo real, como un problema ambiental o social. El objetivo es ayudar a la solución de problemas complejos y que no tienen soluciones sencillas.

Los estudiantes con los que trabajamos en esta investigación suelen mostrar poca disposición y suelen estar poco motivados desde el punto de vista ambiental. Son estudiantes del Grado de Ingeniería de Obras Públicas, una titulación con un enfoque técnico e ingenieril. Además, cursan muy pocas asignaturas de marco ambiental, por lo que suele ser bastante difícil que consigan aprendizajes de manera profunda. Se ha podido comprobar, a lo largo de los años, que estos alumnos, al cursar estas asignaturas relacionadas con el medio ambiente, solo adquieren aprendizajes superficiales y muy fragmentados. Esto se debe en parte a la poca transversalidad que tienen estos contenidos a lo largo de los cursos y a las metodologías de aprendizaje empleadas. Por ejemplo, los trabajos en equipo suelen terminar siendo una suma de trabajos individuales. A todo esto, hay que sumarle la poca motivación que tienen los alumnos por la falta de conciencia de la afección ambiental que sus competencias como futuros ingenieros tienen sobre el medio ambiente. Así, el objetivo es incorporar de manera significativa los valores ambientales en los estudiantes y por lo tanto que sean capaces de influir en su entorno.

\section{Objetivos}

Se desea mejorar la calidad del aprendizaje de los estudiantes, desde el punto de vista de un objetivo global, el desarrollo sostenible, alineado por tanto con los ODS. Se pretende que los estudiantes alcancen un aprendizaje profundo en este ámbito, favoreciendo la transferencia de los conocimientos adquiridos en las diferentes asignaturas a su futura vida profesional y social. Este aprendizaje favorecerá el desarrollo integral del estudiante, no solo desde el punto de vista académico, sino también social y ambiental. Además, se espera conseguir una mayor motivación del estudiante y favorecer el trabajo cooperativo de los estudiantes. Se plantean, por tanto, los objetivos específicos siguientes: 
- Implementar el ABP en las distintas asignaturas

- Incorporar de manera significativa los valores ambientales en los estudiantes

- Determinar si los estudiantes han sido capaces de resolver tareas complejas de manera eficiente

- Valorar si se ha conseguido el aprendizaje colaborativo y cooperativo en los estudiantes

- Evidenciar si se ha conseguido el aprendizaje profundo en los estudiantes

- Valorar si se ha conseguido un mayor interés y motivación del estudiante en las asignaturas

\section{Desarrollo de la innovación}

En esta investigación hemos implantado la metodología de ABP en dos asignaturas del Grado de Ingeniería de Obras Públicas, donde el estudiante es a priori bastante reacio a plantearse que sus futuras obras de ingeniería puedan llegar a afectar al medio ambiente. Las asignaturas son "Ciencia e Impacto Ambiental de la Ingeniería Civil” de $2^{\circ}$ curso y "Evaluación de Impacto Ambiental de la Ingeniería Civil” de $4^{\circ}$ curso. La muestra estudiada coincide con la población total de alumnos que cursan las asignaturas, 42 alumnos. En las dos asignaturas la metodología aplicada es idéntica, el ABP. Se plantea una problemática real a un grupo de alumnos, para cuya solución deben trabajar de forma colaborativa en un proyecto que tienen que diseñar, siguiendo unas pautas iniciales marcadas por el docente, y donde cada alumno tiene un rol individualizado con unos objetivos a conseguir. A pesar de que el docente supervisa continuamente el estado del proyecto, los alumnos trabajan con una total autonomía. Para ello los pasos a seguir en cada asignatura fueron:

- Definición del punto de partida o tema principal

- Formación de equipos colaborativos

- Definición del reto o producto a desarrollar

- Organización y planificación (asignación de roles y definición de tareas y tiempos)

- Búsqueda y recopilación de información

- Análisis y síntesis

- Producción del proyecto

- Presentación del proyecto al resto de compañeros

La evaluación de las estrategias de aprendizaje de los estudiantes se realiza mediante el Cuestionario de Evaluación de las Estrategias de Aprendizaje de los Estudiantes Universitarios (CEVEAPEU) (Gargallo et al., 2009), que ha sido utilizado en diversos estudios. Bustos et al. (2017) lo validan de manera satisfactoria, evaluando la validez factorial, la fiabilidad y las evidencias de validez del cuestionario. El CEVEAPEU tiene en cuenta los aspectos cognitivos y meta-cognitivos relacionados con las estrategias de aprendizaje, y también los factores motivacionales, afectivos y contextuales (Gargallo et al., 2009). Es un buen instrumento, que consta de dos escalas. Por una parte, las estrategias afectivas, de apoyo y de control, y por otra parte las estrategias relacionadas con el procesamiento de la información. Posee seis subescalas (estrategias motivacionales, afectivas, metacognitivas, de control del contexto y otros, de búsqueda y selección de la información, y de procesamiento y uso de la información) y veinticinco estrategias. Consta de 88 ítems y está organizado en dos escalas, seis subescalas y veinticinco estrategias. Está diseñado con el formato de una escala de tipo Likert, con 5 opciones de respuesta, en donde (1) indicaba Muy en Desacuerdo, (2) En Desacuerdo, (3) Indeciso, (4) De Acuerdo y (5) Muy de Acuerdo. Su fiabilidad global es de $\alpha=0.897$.

La Escala I corresponde a las Estrategias afectivas, de apoyo y control (o automanejo) y se divide en 4 subescalas de estrategias:

- Estrategias motivacionales (ESTRMOTIV) que incluye Motivación intrínseca (MOTIN), Motivación extrínseca (MOTEXT), Valor de la tarea (VALTAR), Atribuciones internas 
(ATRINT), Atribuciones externas (ATREXT), Autoeficacia y expectativas (AUTOEFIC) y Concepción de la inteligencia como modificable (CONINT).

- Componentes afectivos (COMPAFEC), que incluye el Estado físico y anímico (ESTFIS) y la Ansiedad (CONTANS).

- Estrategias metacognitivas (ESTRMETACOG), que incluye Conocimiento de objetivos y criterios de evaluación (CONOBJ), Planificación (PLANIF), Autoevaluación (AUTOEV) y Control y autorregulación (CONTAUTOR)

- Estrategias de control del contexto, interacción social y manejo de recursos (ESTRCONTX), que incluye Control del contexto (CRTLCTX) y Habilidades de interacción social y aprendizaje con compañeros (HABSOC)

La Escala II corresponde a las Estrategias relacionadas con el procesamiento de la información, y se divide en 2 subescalas de estrategias:

- Estrategias de búsqueda y selección de información (ESTRBUSINF), que incluye Conocimiento de fuentes y búsqueda de información (CONFUEN) y Selección de información (SELINF)

- Estrategias de procesamiento y uso de la información (ESTRUSOINF), que incluye Adquisición de información (ADQINF), Elaboración (ELABINF), Organización (ORGINF), Personalización y creatividad, pensamiento crítico (PERCRE), Almacenamiento, memorización, uso de recursos mnemotécnicos (ALMEM), Almacenamiento, simple repetición (ALMSR), Transferencia, uso de la información (TRANSF), Manejo de recursos para usar la información adquirida (MANREC)

El esquema metodológico que se siguió fue:

- Al comienzo de la asignatura, los estudiantes contestaron al cuestionario CEVEAPEU (pretest). Para este pretest se solicitó a los estudiantes que contestaran el cuestionario in situ, el primer día de clase y de manera presencial, pensando en su modo de afrontar el aprendizaje general en las asignaturas y materias ya cursadas en la titulación.

- Se aplicó el ABP durante toda la asignatura.

- Tras la asignatura, el último día de clase los estudiantes contestaron de nuevo presencialmente al cuestionario CEVEAPEU (postest). En este caso se pidió que contestaran el cuestionario pensando en su modo de afrontar el aprendizaje en la asignatura en concreto.

- Los resultados obtenidos se analizaron mediante un análisis cualitativo y cuantitativo mediante el paquete estadístico STATGRAPHICS XVII CENTURION.

\section{Resultados}

Tras recopilar los cuestionarios, pretest y postet, se analizó la normalidad de las variables, mediante la prueba de Kolmogorov-Smirnov (K-S), para determinar en qué ítems se podían realizar pruebas paramétricas. Todos los ítems mostraron una distribución normal, por lo que fueron sometidos a un análisis de la varianza (ANOVA) utilizando STATGRAPHICS XVII CENTURION, comparando las puntuaciones obtenidas en el pretest con las del postest. Además, se estimó el tamaño del efecto haciendo uso de la $d$ de Cohen, una de las medidas más empleadas (Morales, 2012). Generalmente, como orientación, suele aceptarse que si $d<0,20$ el tamaño del efecto es pequeño, si $d$ está en torno a 0,5 es moderado, y por encima de 0,8 es grande (Cohen, 1988). Incluso algunos autores (Rosenthal, 1996) indican que si $d>1,30$ el tamaño del efecto es muy grande. Se calcula también $\eta^{2}$, que indica poco efecto si está en torno a 0,01 , en torno a 0,06 indicaría efecto medio y si es superior a 0,14 es ya un efecto grande.

En la tabla 1 se muestra la media, desviación típica, F de Anova, p-value, y el tamaño del efecto ( $d$ de Cohen y $\eta^{2}$ ) para la puntuación global, para cada una de las 2 escalas, 6 subescalas y 25 estrategias. 
Tabla 1. F de ANOVA y significación de las diferentes estrategias de aprendizaje (pretest vs postest) $(N=84 ; G L=1 ; 83)$

\begin{tabular}{|c|c|c|c|c|c|c|c|}
\hline ANOVA & & Media & $\begin{array}{l}\text { Desv } \\
\text { tipica }\end{array}$ & $\mathbf{F}$ & $\begin{array}{c}\text { p- } \\
\text { value }\end{array}$ & $\begin{array}{c}\text { Cohen } \\
\quad d\end{array}$ & $\eta^{2}$ \\
\hline \multirow[t]{2}{*}{ Puntuación global } & Pre & 3,54 & 0,27 & 30,08 & 0,000 & 1,20 & 0,27 \\
\hline & Post & 3,85 & 0,23 & & & & \\
\hline \multirow{2}{*}{$\begin{array}{l}\text { Escala I. Estrategias afectivas, de apoyo y control } \\
\text { (automanejo) }\end{array}$} & Pre & 3,64 & 0,25 & 22,10 & 0,000 & 1,03 & 0,21 \\
\hline & Post & 3,90 & 0,26 & & & & \\
\hline \multirow{2}{*}{$\begin{array}{l}\text { Estrategias motivacionales. } \\
\text { ESTRMOTIV }\end{array}$} & Pre & 3,46 & 0,25 & 57,15 & 0,000 & 1,65 & 0,41 \\
\hline & Post & 3,93 & 0,31 & & & & \\
\hline \multirow{2}{*}{$\begin{array}{l}\text { Motivación intrínseca. } \\
\text { MOTIN }\end{array}$} & Pre & 4,18 & 0,54 & 7,03 & 0,009 & 0,58 & 0,08 \\
\hline & Post & 4,47 & 0,45 & & & & \\
\hline \multirow{2}{*}{$\begin{array}{l}\text { Motivación extrínseca. } \\
\text { MOTEXT }\end{array}$} & Pre & 2,02 & 0,80 & 34,34 & 0,000 & 1,28 & 0,30 \\
\hline & Post & 3,13 & 0,92 & & & & \\
\hline \multirow{2}{*}{$\begin{array}{l}\text { Valor de la tarea. } \\
\text { VALTAR }\end{array}$} & Pre & 4,05 & 0,60 & 3,71 & 0,057 & 0,42 & 0,04 \\
\hline & Post & 4,27 & 0,44 & & & & \\
\hline \multirow{2}{*}{$\begin{array}{l}\text { Atribuciones internas. } \\
\text { ATRINT }\end{array}$} & Pre & 4,03 & 0,52 & 3,02 & 0,086 & 0,38 & 0,04 \\
\hline & Post & 4,22 & 0,45 & & & & \\
\hline \multirow{2}{*}{$\begin{array}{l}\text { Atribuciones externas. } \\
\text { ATREXT }\end{array}$} & Pre & 2,90 & 0,94 & 5,81 & 0,018 & 0,52 & 0,07 \\
\hline & Post & 3,34 & 0,70 & & & & \\
\hline \multirow{2}{*}{$\begin{array}{l}\text { Autoeficacia y expectativas. } \\
\text { AUTOEFIC }\end{array}$} & Pre & 4,13 & 0,44 & 4,38 & 0,039 & 0,46 & 0,05 \\
\hline & Post & 4,34 & 0,50 & & & & \\
\hline \multirow{2}{*}{$\begin{array}{l}\text { Concepción de la inteligencia como } \\
\text { modificable. CONINT }\end{array}$} & Pre & 2,94 & 0,48 & 35,37 & 0,000 & 1,30 & 0,30 \\
\hline & Post & 3,72 & 0,70 & & & & \\
\hline \multirow{2}{*}{$\begin{array}{l}\text { Componentes afectivos. } \\
\text { COMPAFEC }\end{array}$} & Pre & 3,45 & 0,35 & 1,28 & 0,262 & 0,25 & 0,02 \\
\hline & Post & 3,55 & 0,44 & & & & \\
\hline \multirow{2}{*}{$\begin{array}{l}\text { Estado físico y anímico. } \\
\text { ESTFIS }\end{array}$} & Pre & 3,76 & 0,50 & 0,24 & 0,629 & 0,11 & 0,01 \\
\hline & Post & 3,81 & 0,48 & & & & \\
\hline \multirow{2}{*}{$\begin{array}{l}\text { Ansiedad. } \\
\text { CONTANS }\end{array}$} & Pre & 3,15 & 0,58 & 1,17 & 0,283 & 0,24 & 0,01 \\
\hline & Post & 3,30 & 0,64 & & & & \\
\hline \multirow{2}{*}{$\begin{array}{l}\text { Estrategias metacognitivas. } \\
\text { ESTRMETACOG }\end{array}$} & Pre & 3,62 & 0,43 & 18,23 & 0,000 & 0,93 & 0,18 \\
\hline & Post & 3,98 & 0,33 & & & & \\
\hline \multirow{2}{*}{$\begin{array}{l}\text { Conocimiento de objetivos y criterios de } \\
\text { evaluación. CONOBJ }\end{array}$} & Pre & 3,78 & 0,81 & 3,76 & 0,056 & 0,42 & 0,04 \\
\hline & Post & 4,08 & 0,61 & & & & \\
\hline \multirow{2}{*}{$\begin{array}{l}\text { Planificación. } \\
\text { PLANIF }\end{array}$} & Pre & 2,95 & 0,42 & 34,88 & 0,000 & 1,30 & 0,30 \\
\hline & Post & 3,69 & 0,69 & & & & \\
\hline \multirow{2}{*}{$\begin{array}{l}\text { Autoevaluación. } \\
\text { AUTOEV }\end{array}$} & Pre & 3,92 & 0,59 & 3,06 & 0,084 & 0,38 & 0,04 \\
\hline & Post & 4,12 & 0,49 & & & & \\
\hline \multirow{2}{*}{$\begin{array}{l}\text { Control y autoregulación. } \\
\text { CONTAUTOR }\end{array}$} & Pre & 3,83 & 0,49 & 4,20 & 0,044 & 0,45 & 0,05 \\
\hline & Post & 4,03 & 0,37 & & & & \\
\hline
\end{tabular}




\begin{tabular}{|c|c|c|c|c|c|c|c|}
\hline ANOVA & & Media & $\begin{array}{l}\text { Desv } \\
\text { tipica }\end{array}$ & $\mathbf{F}$ & $\begin{array}{c}\text { p- } \\
\text { value }\end{array}$ & $\begin{array}{c}\text { Cohen } \\
d\end{array}$ & $\eta^{2}$ \\
\hline \multirow{2}{*}{$\begin{array}{l}\text { Estrategias de control del contexto, interacción } \\
\text { social y manejo de recursos. ESTRCONTX }\end{array}$} & Pre & 4,00 & 0,43 & 1,94 & 0,168 & 0,30 & 0,02 \\
\hline & Post & 4,13 & 0,42 & & & & \\
\hline \multirow{2}{*}{$\begin{array}{l}\text { Control del contexto. } \\
\text { CRTLCTX }\end{array}$} & Pre & 3,91 & 0,56 & 1,78 & 0,186 & 0,29 & 0,02 \\
\hline & Post & 4,07 & 0,50 & & & & \\
\hline \multirow{2}{*}{$\begin{array}{l}\text { Habilidades de interacción social y } \\
\text { aprendizaje con compañeros. HABSOC }\end{array}$} & Pre & 4,09 & 0,56 & 0,78 & 0,378 & 0,19 & 0,01 \\
\hline & Post & 4,20 & 0,52 & & & & \\
\hline \multirow{2}{*}{$\begin{array}{l}\text { Escala II. Estrategias relacionadas con el } \\
\text { procesamiento de la información }\end{array}$} & Pre & 3,45 & 0,35 & 24,52 & 0,000 & 1,08 & 0,23 \\
\hline & Post & 3,79 & 0,29 & & & & \\
\hline \multirow{2}{*}{$\begin{array}{l}\text { Estrategias de búsqueda y selección e } \\
\text { información. ESTRBUSINF }\end{array}$} & Pre & 3,42 & 0,44 & 12,22 & 0,001 & 0,76 & 0,13 \\
\hline & Post & 3,75 & 0,42 & & & & \\
\hline \multirow{2}{*}{$\begin{array}{l}\text { Conocimiento de fuentes y búsqueda de } \\
\text { información. CONFUEN }\end{array}$} & Pre & 3,30 & 0,66 & 5,71 & 0,019 & 0,52 & 0,06 \\
\hline & Post & 3,65 & 0,64 & & & & \\
\hline \multirow{2}{*}{$\begin{array}{l}\text { Selección de información. } \\
\text { SELINF }\end{array}$} & Pre & 3,53 & 0,46 & 10,39 & 0,002 & 0,70 & 0,11 \\
\hline & Post & 3,85 & 0,45 & & & & \\
\hline \multirow{2}{*}{$\begin{array}{l}\text { Estrategias de procesamiento y uso de la } \\
\text { información. ESTRUSOINF }\end{array}$} & Pre & 3,48 & 0,34 & 27,56 & 0,000 & 1,14 & 0,25 \\
\hline & Post & 3,84 & 0,28 & & & & \\
\hline \multirow{2}{*}{$\begin{array}{l}\text { Adquisición de información. } \\
\text { ADQINF }\end{array}$} & Pre & 3,98 & 0,49 & 2,66 & 0,107 & 0,35 & 0,03 \\
\hline & Post & 4,13 & 0,35 & & & & \\
\hline \multirow{2}{*}{$\begin{array}{l}\text { Elaboración de información. } \\
\text { ELABINF }\end{array}$} & Pre & 2,92 & 0,64 & 17,79 & 0,000 & 0,92 & 0,18 \\
\hline & Post & 3,52 & 0,66 & & & & \\
\hline \multirow{2}{*}{$\begin{array}{l}\text { Organización de información. } \\
\text { ORGINF }\end{array}$} & Pre & 3,89 & 0,56 & 3,85 & 0,053 & 0,43 & 0,05 \\
\hline & Post & 4,10 & 0,41 & & & & \\
\hline \multirow{2}{*}{$\begin{array}{l}\text { Personalización y creatividad, } \\
\text { pensamiento crítico. PERCRE }\end{array}$} & Pre & 3,68 & 0,51 & 9,18 & 0,003 & 0,66 & 0,10 \\
\hline & Post & 4,00 & 0,46 & & & & \\
\hline \multirow{2}{*}{$\begin{array}{l}\text { Almacenamiento, memorización, uso de } \\
\text { recursos mnemotécnicos. ALMEM }\end{array}$} & Pre & 3,28 & 1,01 & 4,02 & 0,048 & 0,44 & 0,05 \\
\hline & Post & 3,67 & 0,76 & & & & \\
\hline \multirow{2}{*}{$\begin{array}{l}\text { Almacenamiento, simple repetición. } \\
\text { ALMSR }\end{array}$} & Pre & 2,37 & 0,94 & 13,16 & 0,001 & 0,79 & 0,14 \\
\hline & Post & 3,09 & 0,90 & & & & \\
\hline \multirow{2}{*}{$\begin{array}{l}\text { Transferencia, uso de la información. } \\
\text { TRANSF }\end{array}$} & Pre & 4,02 & 0,52 & 5,73 & 0,019 & 0,52 & 0,07 \\
\hline & Post & 4,29 & 0,52 & & & & \\
\hline \multirow{2}{*}{$\begin{array}{l}\text { Manejo de recursos para usar la } \\
\text { información adquirida. MANREC }\end{array}$} & Pre & 3,70 & 0,66 & 1,48 & 0,227 & 0,27 & 0,02 \\
\hline & Post & 3,88 & 0,75 & & & & \\
\hline
\end{tabular}

En las figuras 1, 2 y 3 se muestran las diferencias existentes entre las medias de las distintas variables en el pretest y en el postest. Se observa que para todas las variables, las medias son superiores en el postest. 


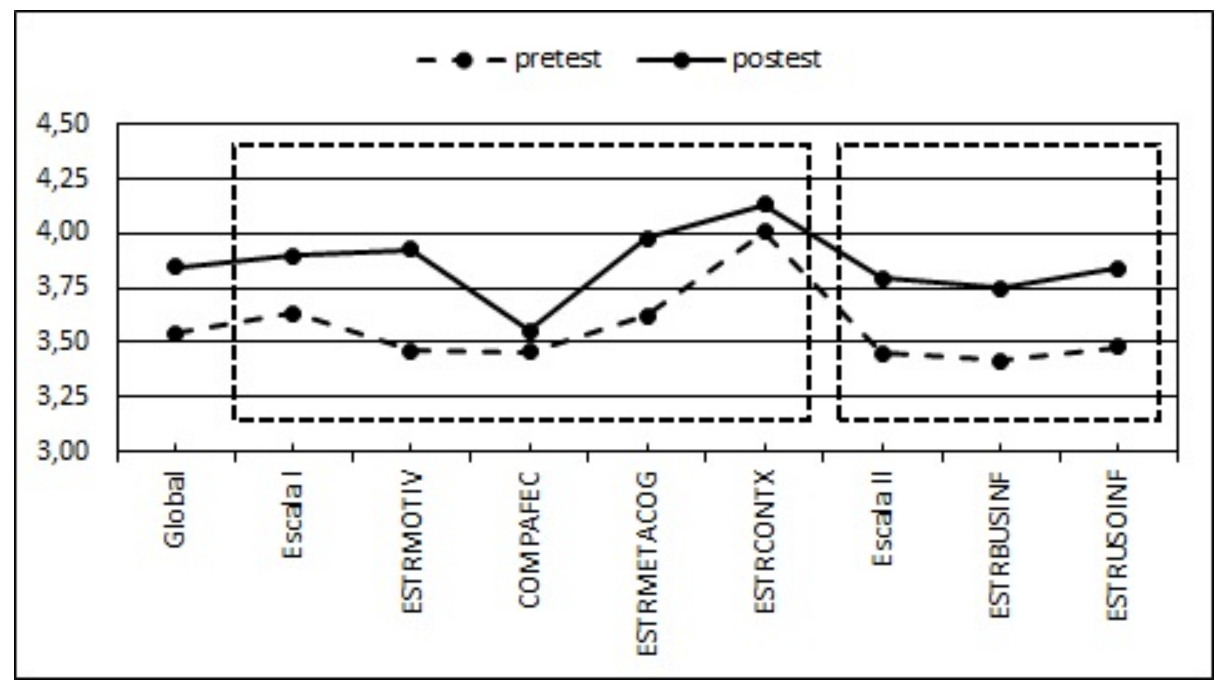

Fig. 1 Medias de Puntuación global, Escalas y Subescalas en pretest y postest

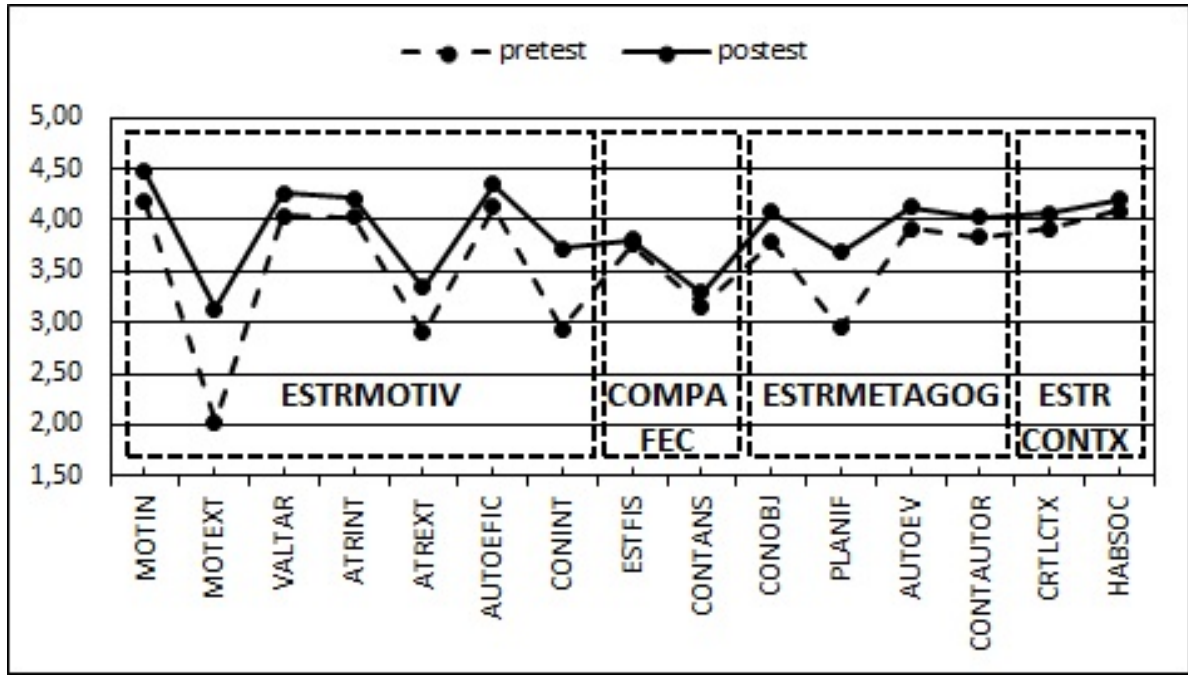

Fig. 2. Medias de las Estrategias de la Escala I en pretest y postest

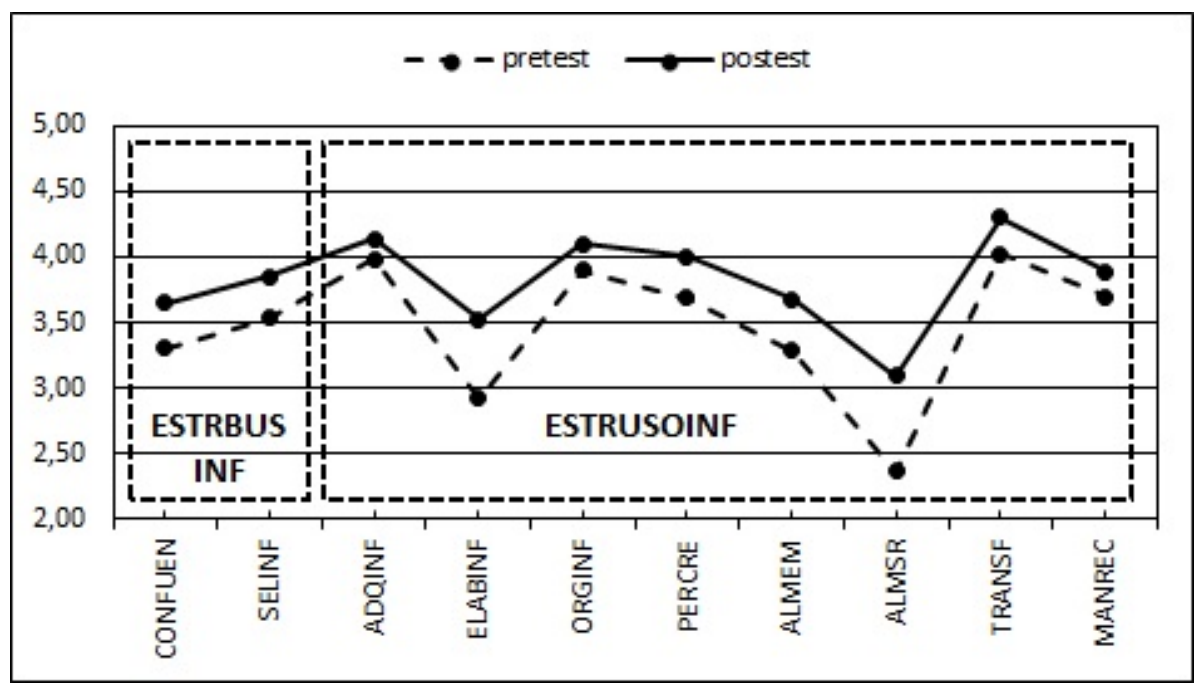

Fig. 3. Medias de las Estrategias de la Escala II en pretest y postest

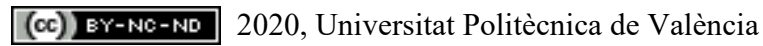

Congreso In-Red (2020) 
Se encontraron diferencias estadísticamente significativas del pretest al postest en la puntuación media global del cuestionario, con mejora en el postest $\left[\mathrm{F}(1,83)=30,08\right.$, $\mathrm{p}$-value $<0,001, d$-cohen $=1,20, \eta^{2}=$ $0,27]$, con un tamaño del efecto grande.

También se encontraron diferencias estadísticamente significativas del pretest al postest en la primera escala, de estrategias afectivas, de apoyo y control $\left[\mathrm{F}(1,83)=22,10\right.$, $\mathrm{p}$-value $<0,001, d$-cohen $=1,03, \eta^{2}=$ $0,21]$, con un tamaño del efecto grande, y mejorando las puntuaciones en el postest. Estas estrategias se dividen en 4 subescalas, que aunque no van dirigidas al procesamiento de los materiales, son absolutamente necesarias para un aprendizaje de calidad. Son las estrategias que ponen en marcha el proceso, ayudan a mantener el esfuerzo cognitivo, movilizan y controlan la parte afectiva, ayudan a manejar el contexto, y permiten la planificación, evaluación y control de la propia actividad en el aprendizaje (Gargallo et al., 2015).

Se dieron diferencias estadísticamente significativas del pretest al postest en dos de las cuatro subescalas: estrategias motivacionales $\left[\mathrm{F}(1,83)=57,15, \mathrm{p}\right.$-value $<0,001, d$-cohen $\left.=1,65, \eta^{2}=0,41\right]$, estrategias metacognitivas $\left[\mathrm{F}(1,83)=18,23\right.$, p-value $<0,001, d$-cohen $\left.=0,93, \eta^{2}=0,18\right]$. En ambos casos el tamaño del efecto fue grande y mejoraron las puntuaciones en el postest. En las otras dos escalas no existieron diferencias estadísticamente significativas, aunque se obtuvo mayor valoración en el postest tanto para los Componentes afectivos como para las Estrategias de control del contexto, interacción social y manejo de recursos.

En el siguiente nivel, en el caso de las estrategias, se encontraron diferencias significativas en 7 de las 15 .

Así, dentro de las Estrategias motivacionales (ESTRMOTIV), que se encargan de explorar el tipo de motivación para aprender (intrínseca - extrínseca) del estudiante, la Motivación intrínseca, Motivación extrínseca, Atribuciones externas, Autoeficacia y expectativas y Concepción de la inteligencia como modificable poseen diferencias estadísticamente significativas, con valoraciones mayores en el postest y tamaños del efecto medios o grandes. Las estrategias motivacionales de Valor de la tarea y de Atribuciones internas, a pesar de que en ambas se obtuvieron valoraciones mayores en el postest, no muestran diferencias estadísticamente significativas.

Ninguna de las dos estrategias incluidas en los Componentes afectivos, Estado físico y anímico y Ansiedad (COMPAFEC), que exploran el estado físico y anímico del estudiante, mostraron diferencias significativas, a pesar de que en ambas se obtuvieron valoraciones mayores en el postest.

Dentro de las Estrategias metacognitivas (ESTRMETACOG), que exploran los puntos fuertes y débiles del universitario ante el aprendizaje, la Planificación y el Control y autorregulación muestran diferencias estadísticamente significativas, con valoraciones mayores en el postest y tamaños del efecto medios o grandes. El Conocimiento de objetivos y criterios de evaluación y la Autoevaluación no muestran diferencias significativas, aunque obtienen mayor valoración en el postest.

Ninguna de las dos estrategias de Control del contexto y Habilidades de interacción social y aprendizaje con compañeros (ESTRCONTX), que examinan las condiciones de estudio del estudiante y analizan el trabajo que realiza con otros para mejorar el aprendizaje, mostraron diferencias significativas.

Las diferencias también fueron significativas en la segunda escala, que corresponde a las estrategias relacionadas con el procesamiento de la información, dirigidas a la adquisición, elaboración, organización y almacenamiento de la información $\left[\mathrm{F}(1,83)=24,52\right.$, p-value $<0,001, d$-cohen $\left.=1,08, \eta^{2}=0,23\right]$, con un valor del tamaño del efecto también grande, y mejorando las puntuaciones en el postest.

Se obtuvieron diferencias estadísticamente significativas del pretest al postest en las dos subescalas: estrategias de búsqueda y selección de información $\left[\mathrm{F}(1,83)=12,22\right.$, p-value $<0,005, d$-cohen $=0,76, \eta^{2}=$ 
$0,13]$ y estrategias de procesamiento y uso de la información $[\mathrm{F}(1,83)=27,56$, p-value $<0,001, d$-cohen $=$ $\left.1,14, \eta^{2}=0,25\right]$. En todos los casos el tamaño del efecto fue grande y mejoraron las puntuaciones en el postest.

En el siguiente nivel, en el caso de las estrategias, se encontraron diferencias significativas en 7 de las 10 .

De las estrategias relacionadas con la búsqueda y selección de información (ESTRBUSINF), que exploran las acciones cotidianas para acercarse a la información a aprender, y los mecanismos para discriminar lo importante de lo no importante, tanto la Selección de información como el Conocimiento de fuentes y búsqueda de información mostraron diferencias estadísticamente significativas con mayores valoraciones en el postest.

De todas las Estrategias de procesamiento y uso de la información (ESTRUOINF), que exploran las acciones que realiza el alumno para adquirir, codificar y organizar la información, los mecanismos de memorización y la capacidad para transferir y utilizar la información aprendida, 5 de ellas mostraron diferencias estadísticamente significativas. Son Elaboración de información; Personalización y creatividad y pensamiento crítico; Almacenamiento, memorización y uso de recursos mnemotécnicos; Almacenamiento, simple repetición; y Transferencia y uso de la información. Todas ellas poseen mayores valoraciones en el postest. En cambio las estrategias de Adquisición de información, Organización de información y Manejo de recursos para usar la información adquirida no muestran diferencias significativas, a pesar de que obtuvieron mayor valoración en el postest.

Estos resultados dejan patente que la metodología utilizada (ABP) mejora de manera significativa las estrategias de aprendizaje de los estudiantes, fundamentalmente en las estrategias motivacionales, en las estrategias metacognitivas, en las de búsqueda y selección de información y en las de procesamiento y uso de la información. Sin embargo, hay dos estrategias que, a pesar de mejorar, no lo hacen de manera significativa. Son las relacionadas con los componentes afectivos y el control del contexto.

\section{Conclusiones}

Los resultados de nuestro trabajo muestran que con la metodología aplicada, el Aprendizaje Basado en Proyectos, los estudiantes utilizan más y mejores estrategias de aprendizaje.

Se obtuvieron diferencias significativas en las estrategias de aprendizaje de los estudiantes, en la puntuación global, en las dos escalas y en cuatro de las seis subescalas: Estrategias motivacionales, Estrategias metacognitivas, Estrategias de Búsqueda y selección de información y Estrategias de Procesamiento y uso de la información.

Las diferencias fueron estadísticamente significativas en catorce estrategias: Motivación intrínseca, Motivación extrínseca, Atribuciones externas, Autoeficacia y expectativas, Concepción de la inteligencia como modificable, Planificación, Control y autoregulación, Conocimiento de fuentes y búsqueda de información, Selección de información, Elaboración de información, Personalización y creatividad (pensamiento crítico), Almacenamiento y Transferencia y uso de la información. En el resto de estrategias las diferencias no fueron estadísticamente significativas, pero todas mejoraron en el postest.

Las estrategias que mejoran en mayor medida tras la aplicación del Aprendizaje Basado en Proyectos son las estrategias motivacionales (fundamentalmente la motivación extrínseca y la concepción de la inteligencia como modificable), las estrategias metacognitivas (sobre todo la planificación), las estrategias de búsqueda de información (fundamentalmente la selección de información) y las estrategias de procesamiento y uso de la información (fundamentalmente la elaboración). Las estrategias que menos mejoran tras la aplicación del ABP son las correspondientes a los componentes afectivos (estado físico y anímico y ansiedad) y las estrategias de control del contexto, interacción social y manejo de recursos. 
Los resultados obtenidos son bastante relevantes, debido a que el uso de metodologías activas centradas en el aprendizaje, mejora en gran medida las estrategias de aprendizaje. Principalmente son las estrategias Motivacionales las que mejoran, lo que confirma que el uso de la metodología aplicada hace mejorar la motivación de los estudiantes. También las estrategias Metacognitivas mejoran en gran medida, lo que está altamente relacionado con el trabajo exigido a los estudiantes, que necesitan este tipo de habilidades. Del mismo modo también mejoran todas las estrategias relacionadas con el procesamiento de la información, que son imprescindibles para llevar a cabo con éxito el aprendizaje.

La utilización de esta metodología como herramienta pedagógica ha conseguido que los estudiantes aprendan mejor, tanto de forma individual como en grupo. Esta metodología ha exigido una participación activa, constante y cooperativa de los estudiantes, que han tenido más responsabilidad en el desarrollo de su trabajo y se han acercado a la realidad de su futuro profesional.

\section{Referencias}

ANTONelli, C. (2017). “Cómo incentivar el aprendizaje profundo?” en XXV Jornadas de Reflexión Académica en Diseño y Comunicación, vol. 30, p. 133-135.

BIGGS, J.B. y TANG, C. (2007). Teaching for Quality Learning at University, 3rd edit. Glasgow: Open University Press.

Bustos, V., Oliver, A., Galiana, L. y Sancho, P. (2017). "Propiedades psicométricas del CEVEAPEU: Validación en población peruana" en Educación XX1, vol. 20, issue 1, p. 299-318. <https://doi.org/10.5944/educxx1.17513> [Consulta: 15 de enero de 2020]

Castro, A., Lopez, G., Padilla A.L., Melendez, L. y Escobedo, A.B. (2018). "La investigación de un proyecto usado como estrategia para valorar el impacto ambiental por alumnos de química" en Revista Electrónica sobre Cuerpos Académicos y Grupos de Investigación, vol. 5, issue 10, p. 1-14.

CoHEn, J. (1988). Statistical Power Analysis for the Behavioral Sciences. 2nd. edit., Hillsdale, N.J. : Lawrence Erlbaum Associates.

Diaz-Mujica, A. y Perez-Villalobos, M.V. (2013). "Autoeficacia, enfoque de aprendizaje profundo y estrategias de aprendizaje" en International Journal of Developmental and Educational Psychology, INFAD Revista de Psicologia, vol. 2, issue 1, p. 341-346.

EntwistLe, N. (2009). Teaching for Understanding at University: Deep Approaches and Distinctive Ways of Thinking. Hampshire \& New York: Palgrave Macmillan.

FASCE, E. (2007). "Aprendizaje profundo y superficial, Tendencias y perspectivas" en Rev. Educ. Cienc. Salud, vol. 4, issue 1 , p. 7-8.

Fernandez, C. y Arquero, J.L. (2011). "Evaluación de innovaciones y enfoques de aprendizaje. Presentación preliminar de un instrumento de medida. Innovations assessment and approaches to learning. Preliminar presentation of a questionnaire" en IV Jornadas de Innovación e Investigación Docente. Sevilla: Edicion Digital Atres. 214-225. Disponible en $<\mathrm{http}: /$ hdl.handle.net/1441/43202> [Consulta: 9 de enero de 2020]

Gargallo, B., Morera, I. y Garcia, E. (2015). "Metodología innovadora en la universidad. Sus efectos sobre los procesos de aprendizaje de los estudiantes universitarios" en Anales de psicología, vol. 31, issue 3, p. 901-915. $<$ http://dx.doi.org/10.6018/analesps.32.1.179871> [Consulta: 20 de septiembre de 2019]

Gargallo, B., Suarez-Rodriguez, J.M. y Perez, C. (2009). "El cuestionario CEVEAPEU. Un instrumento para la evaluación de las estrategias de aprendizaje de los estudiantes universitarios" en Relieve, vol. 15, issue 2, p. 1-31.

KATZ, L.G. y ChARD, S.C. (1989). Engaging children's minds: The Project approach. Norwood, NJ: Ablex.

Lopez-Aguado, M. y Gutierrez-Provecho, L. (2014). "Modelo explicativo del efecto de los enfoques de aprendizaje sobre el rendimiento y el papel modulador de la dedicación temporal" en Revista de Investigación Educativa, vol. 32, issue 2, p. 447-462. <http://dx.doi.org/10.6018/rie.32.2.164761> [Consulta: 15 de diciembre de 2019] 
Lopez-Aguado, M. y Lopez-Alonso, A.I. (2013). "Los enfoques de aprendizaje. Revisión conceptual y de investigación //Learning Approaches: Theoretical and Research Review" en Revista Colombiana de Educación, vol. 64, p. 131-153.

MALdonado, M. (2008). "Aprendizaje basado en proyectos colaborativos. Una experiencia en educación superior" en Laurus, vol. 14, issue 28, p. 158-180.

Marti, J.A., Heydrich, M., Rojas, M. y Hernandez, A. (2009). "Aprendizaje basado en proyectos: una experiencia de innovación docente" en Revista Universidad EAFIT, vol. 46, issue 158, p. 11-21.

Ministerio de Asuntos Exteriores, Union Europea y CoOperacion, Secretaria de Estado de CoOperacion InTERnACIONAL y PARA IBEROAMERICA Y El CARIBE, DirecCiOn GENERAL DE Politicas de Desarrollo Sostenible. (2018). "Plan de Acción para la Implementación de la Agenda 2030. Hacia una Estrategia Española de Desarrollo Sostenible" NIPO (en línea): 108-19-003-7 <http://transparencia.gob.es> [Consulta: 29 de mayo de 2019]

Morales, P. (2012). "El tamaño del efecto (effect size): análisis complementarios al contraste de medias". $<$ http://web.upcomillas.es/personal/peter/investigacion/TamañoDelEfecto.pdf > [Consulta: 14 de julio de 2019]

RAMSDEN, P. (2003). Learn to teach in Higher Education. Nueva York: Routledge.

RockSTRÖM, J. y SuKHDEV, P. (2016). "Sustainable development goals. How food connects all the SDGs". Stockholm Resilience Centre. Stockholm University < https://www.stockholmresilience.org/> [Consulta : 29 de mayo de 2019]

Rodriguez-Sandoval, E., Vargas-Solano, E.M. y LunA-Cortes, J. (2010). "Evaluación de la estrategia "aprendizaje basado en proyectos" en Educación y Educadores, vol. 13, issue 1, p. 13-25.

Rosenthal, J.A. (1996). "Qualitative descriptors of strength of association and effect size" en Journal of Social Service Research, vol. 21, issue 4, p. 37-59. 